\title{
Tempo de permanência no transporte escolar sobre o desempenho estudantil
}

\author{
GOULART, Lívia Mara Lima' \\ MORA/S, Alaine Andrade de ${ }^{2}$ \\ VIEIRA JR, Nilton ${ }^{3}$
}

\section{RESUMO}

Nas comunidades rurais, o transporte escolar é fundamental no acesso à unidade de ensino, porém o tempo que os estudantes permanecem no veículo até as escolas acaba sendo muito longo, o que acarreta no cansaço e desgaste dos alunos e, consequentemente, influencia no seu rendimento escolar. Um exemplo desta situação acontece no Instituto Federal de Roraima/campus Novo Paraíso, localizado na zona rural de Caracaraí-RR, onde o tempo de deslocamento até a escola é maior que o recomendado e o desempenho dos alunos no Exame Nacional do Ensino Médio nos últimos dois anos foi abaixo da média nacional. Para minimizar esse problema, é necessário, além de um melhor planejamento das rotas do transporte rural, políticas públicas para melhorar as vias rurais e um plano pedagógico de ensino que seja mais atrativo e retrate a realidade dos alunos do campo.

Transporte escolar rural. Desempenho escolar. Tempo de deslocamento.

\section{Time length of stay in school transport on student performance}

\begin{abstract}
In rural communities, school transport is essential for access to the teaching unit, but the time students spend in the vehicle until school are very long, which results in tiredness and emotional distress, consequently, influences their school performance. An example of this situation is at the Roraima Federal Institute / Novo Paraíso campus, located in rural Caracaraí-RR, where the travel time to school is longer than recommended and student performance in the National High School Exam in the last two years was below the national average. To minimize this problem, better planning of rural transport routes,
\end{abstract}

\footnotetext{
${ }^{1}$ Doutora em Ciência florestal e Mestrado em Ciência Florestal pela Universidade Federal de Viçosa pela Faculdade de Ciências Agronômicas da UNESP. Professora do Instituto Federal de Roraima IFRR. Email: livia.goulart@ifrr.edu.br. Lattes: Lattes: http://lattes.cnpq.br/2811724270326388. ORCID: https://orcid.org/0000-0002-6004-4545.

${ }^{2}$ Mestre em Engenharia de produção. Pós-graduada em Mídias na Educação pela UFRR. Professora EBTT do Instituto Federal de Educação, Ciência e Tecnologia de Roraima, campus Novo Paraíso. Lattes: http://lattes.cnpq.br/0552163685334278. ORCID: https://orcid.org/0000-0002-3113-7283.

${ }^{3}$ Doutor em Engenharia Elétrica. Professor Associado III do Instituto Federal de Minas Gerais IFMG. ORCID: http://orcid.org/0000-0003-1077-8302.
}

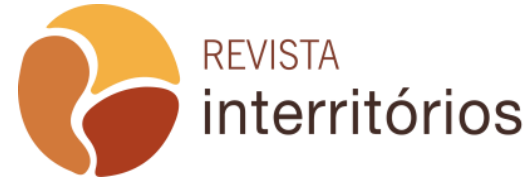

Interritórios | Revista de Educação Universidade Federal de Pernambuco, Caruaru, BRASIL | V.5 N.9 [2019] 
public policies to improve rural roads and a more attractive pedagogical teaching plan that reflects the reality of rural students are needed.

Rural school transportation. School performance. Travel time.

\section{La durata della permanenza nel trasporto scolastico sull reddito scolare degli studenti}

\section{RIASSUNTO}

Nelle comunità rurali, il trasporto scolastico è essenziale per l'accesso all'unità didattica, ma il tempo che gli studenti trascorrono nel veicolo fino a quando la scuola finisce per essere molto lungo, il che si traduce in stanchezza degli studenti e, di conseguenza, influenza nel loto reddito scolare. Ne è un esempio I'Istituto Federale di Roraima / Novo Paraíso, nel campus rurale di Caracaraí$\mathrm{RR}$, dove il tempo di percorrenza è più lungo di quanto raccomandato e la performance degli studenti al Exame Nazionale de le Superiori negli ultimi due anni era inferiore alla media nazionale. Al fine di ridurre al minimo questo problema, è necessario, oltre a una migliore pianificazione delle rotte di trasporto rurale, politiche pubbliche per migliorare le strade rurali e un piano di insegnamento che sia più attraente e rappresenti la realtà degli studenti rurali.

Trasporto Scolastico Rurale, Rendimento Scolastico, Tempo di Percorrenza.

\section{Duración de la estadía en el transporte escolar por el desempeño del estudiante}

\section{RESUMEN}

En las comunidades rurales, el transporte escolar es fundamental en el acceso a unidad de enseñanza pero el tiempo que los estudiantes pasan en el vehículo incluso las escuelas terminan siendo muy largas, lo que provoca cansancio y desgaste en consecuencia influye en su rendimiento escolar. Uno ejemplo de esta situación ocurre en el Instituto Federal de Roraima / campus Novo Paraíso, ubicado en la zona rural de Caracaraí-RR, donde el tiempo de viajar a la escuela es más alto de lo recomendado y el desempeño de los estudiantes en el examen nacional de la escuela secundaria en los últimos dos años han estado por debajo del promedio nacional. Para minimizar este problema, es necesario, además de un mejor planificación de rutas de transporte rural, políticas públicas para mejorar caminos rurales y un plan de enseñanza más atractivo y retrata la realidad de los estudiantes de campo.

Autobús escolar rural. Rendimiento escolar. Tiempo de viaje. 


\section{INTRODUÇÃO}

$\mathrm{Na}$ zona rural, existem grandes dificuldades em vários aspectos, como diferenças salariais e níveis de consumo, além do fornecimento inadequado de serviços essenciais como saúde, água, esgoto, escolas e transporte (PEGORETTI; SANCHES, 2006). Na área da educação, um dos principais problemas é o acesso dos alunos às escolas rurais e seu longo período de permanência nos veículos de transporte.

A população rural tem mais dificuldades no acesso às unidades de ensino, em geral, devido às grandes distâncias a serem percorridas. Assim, o transporte escolar torna-se fundamental no acesso à unidade de ensino e, em sua maioria, o transporte escolar gratuito fornecido pelo poder público representa a única forma que o aluno das zonas rurais dispõe para chegar à escola.

Egami et al., (2015) afirma que para os estudantes residentes na área rural, o transporte torna-se essencialmente importante para que se consiga ter acesso à escola. Porém, chegar até a escola do campo é um grande desafio para seus estudantes e corpo docente devido às dificuldades em função da distância, falta de transporte e estradas inadequadas, dentre outros fatores. Tal fato somente faz aumentar o abismo da qualidade e da aprendizagem entre escolas rurais e urbanas.

Em se tratando do transporte rural, o transporte escolar é o segmento que gera o maior número de viagens diárias. Segundo o Centro Interdisciplinar de Estudos em Transportes (CEFTRU), o transporte escolar rural é o transporte coletivo de estudantes que residem e/ou estudam na zona rural, para fins educacionais, entre sua residência e uma instituição educacional, em horários previamente estabelecidos (CEFTRU, 2008).

Com intuito de diminuir as dificuldades dos alunos de áreas rurais em acessar a escola, a Lei $n^{\circ} 10.709 / 2003$ estabeleceu que os Estados passariam a assumir o transporte escolar dos alunos da rede estadual e responsabilizou os Municípios pelo transporte dos alunos da rede municipal. Além disso, programas federais de apoio ao transporte escolar foram implementados, como o Programa Nacional de Transporte Escolar (PNTE), criado em 1994, sendo o primeiro programa destinado diretamente ao transporte dos alunos no trajeto residência-escola-residência, auxiliando na compra de veículos novos. Dez anos depois, em 2004, o Programa Nacional de Apoio ao Transporte Escolar substituiu o PNTE e teve como objetivo oferecer ajuda financeira aos Municípios para o transporte de alunos das áreas rurais para a escola (ARAÚJO, 2008).

Com esse panorama, o transporte escolar rural vem crescendo em 
importância, a ponto de se tornar um processo complementar a educação, apto a contribuir para o acesso e a permanência dos estudantes residentes em áreas rurais na escola (FRIGATO, 2016; MORAIS, 2018).

\section{Tempo de permanência no transporte escolar rural e o rendimento escolar dos alunos}

Segundo Câmara et al., (2008), o tempo de viagem até a escola influência de forma direta no conforto escolar do aluno, uma vez que gera desgaste físico e emocional. Assim, uma vez garantidos os parâmetros de segurança, quanto menor o tempo de viagem, maior o conforto e a praticidade para o usuário do transporte escolar rural. Um longo trajeto para escola tornase cansativo e desgastante, fazendo com que o aluno não assista as aulas de forma adequada, prejudicando a apreensão dos conteúdos repassados pelo professor e, consequentemente, influenciando seu rendimento escolar (MARTINS, 2010).

Em condições normais, o tempo máximo de viagem entre o ponto de embarque e a escola deve ser de 45 minutos (PEGORETTI, 2005), não sendo recomendado mais que uma hora (GUIMARÃES, 2004) em um percurso (ida ou volta), totalizando duas horas diárias de tolerância no transporte escolar. Em decorrência das más condições das vias rurais, o tempo de viagem entre o ponto de embarque e a escola tende a elevar-se. A existência de vias não pavimentadas, que se deterioram com o período chuvoso, dificulta ainda mais o deslocamento de veículos e pedestres (SANCHES e FERREIRA, 2017). Períodos longos de viagem provocam cansaço, aborrecimento e queda da produtividade, que podem afetar no aprendizado do aluno (PEGORETTI, 2005).

Sobre o tempo gasto pelos alunos no transporte escolar, nota-se, de maneira geral que, quanto maior é esse tempo, pior é o efeito sobre os rendimentos dos alunos, pois além do cansaço ao chegar na escola, o tempo despendido no deslocamento implica em redução do tempo dedicado aos estudos extraclasse, atividades familiares e de lazer (MARTINS, 2006).

\section{Desempenho no Exame Nacional do Ensino Médio e o tempo gasto no transporte escolar}

Como exposto anteriormente, o tempo gasto no transporte escolar rural pode influenciar significativamente no desempenho acadêmico dos alunos. Um exemplo dessa situação acontece no Instituto Federal de Educação, Ciência e 
Tecnologia de Roraima, Campus Novo Paraíso (IFRR/CNP), que é uma unidade de ensino federal criado pela Lei Federal no 11.892/2008, instalado estrategicamente na zona rural do município de Caracaraí, em uma área territorial de $99.325,70 \mathrm{~km}^{2}$, às margens da BR 174 que interliga o Estado de Roraima ao Estado de Amazonas, a $12 \mathrm{~km}$ do anel rodoviário da Vila Novo Paraíso.

Essa unidade do IFRR foi construída com finalidade de atender várias cidades do seu entorno, bem como a população rural dessas localidades, com vistas a oportunizar o desenvolvimento socioeconômico da região sul do Estado de Roraima. O IFRR/CNP também é acessado pela BR 210, interligando-se aos municípios de São Luiz do Anauá, São João da Baliza e Caroebe, e pela BR 432 que permite o acesso ao município do Cantá. Como está localizado fora do perímetro urbano e por ofertar Ensino Médio na última etapa da Educação Básica, caracteriza-se como escola rural.

O IFRR/CNP oferta para trezentos e trinta e cinco alunos educação básica, profissional e tecnológica por meio dos Cursos Técnicos de Agroindústria, Agropecuária Integrados ao Ensino Médio e na Modalidade Alternância, além de ensino superior, o curso de Bacharelado em Agronomia.

A distância percorrida diariamente pelos discentes do IFRR/CNP na ida e na volta da instituição varia entre $96 \mathrm{~km}$ e $226 \mathrm{~km}$, com tempo médio percurso entre $2 \mathrm{~h} 14 \mathrm{~min}$ a $4 \mathrm{~h} 30 \mathrm{~min}$ (Tabela 1 ), sendo o trajeto feito por meio de ônibus fretados (contratados) (MORAIS, 2018). Assim, problemática existente no transporte no ambiente rural é evidenciada nessa instituição, pois os trajetos são longos e, na maioria das vezes, únicos, ou seja, há poucas alternativas de caminhos para chegar até 0 aluno, bem como as condições das estradas costumam ser precárias, aumentando o tempo de viagem e os custos dos veículos.

Tabela 1: Informações sobre as rotas e preços dos ônibus fretados pelo IFRR/CNP para atendimento dos alunos.

\begin{tabular}{l|c|c}
\hline Rotas do ônibus escolar & $\begin{array}{c}\text { Total Percurso (Ida e } \\
\text { volta) - Km }\end{array}$ & $\begin{array}{c}\text { Duração da viagem } \\
\text { (Ida e volta) - Horas }\end{array}$ \\
\hline Caracaraí/BR174 & 226 & 4h30min \\
\hline Caroebe/São Luís do Anauá & 212 & $3 \mathrm{~h} 55 \mathrm{~min}$ \\
\hline Vila Nova Colina BR 174 & 184 & $2 \mathrm{~h} 14 \mathrm{~min}$ \\
\hline São João do Baliza/BR 210 & 158 & $3 \mathrm{~h} 35 \mathrm{~min}$ \\
\hline Vila Baraúana km 55/Novo Paraíso BR 174 & 124 & $2 \mathrm{~h} 38 \mathrm{~min}$ \\
\hline Rorainópolis/ BR 174 & 96 & $2 \mathrm{~h} 35 \mathrm{~min}$ \\
\hline
\end{tabular}

Fonte: Morais (2018). 
Pela Tabela 1, observa-se que, em cidades como Rorainópolis e Vila Baraúana km 55/Novo Paraíso BR 174, apesar de serem as localidades mais próximas ao IFRR/CNP, gastam mais tempo no percurso de ida e volta que em cidades mais longínquas, como Vila Nova Colina BR 174 (184 km de percurso total), devido às excessivas paradas e demora para embarque e desembarque de alunos.

Apesar do IFRR/CNP contar com uma estrutura adequada para atender o número de alunos matriculados e veículos de transporte dentro das normas de segurança e com conforto, o desempenho dos alunos do ensino médio nos dois últimos anos na prova do Exame Nacional do Ensino Médio (Enem) foram abaixo da média nacional, conforme representado na Tabela 2. Ao comparar as notas do Enem de 2017 e 2018 dos alunos do IFRR/CNP com o dos alunos do IFRR/campus Boa Vista (IFRR/BV) (Tabela 2) que está localizado no bairro Pricumã, na capital de Roraima, fica evidente a discrepância das notas. Como as principais diferenças entre os dois campus do Instituto Federal de Roraima são a localização e o tempo que os alunos gastam no percurso até a escola, pode-se inferir que uma das causas do baixo rendimento dos alunos do IFRR/CNP no Enem nos dois últimos anos seria o longo tempo de permanência dentro do veículo, aspecto que pode contribuir para o baixo desempenho escolar, repetência e a evasão. Outra consequência importante a ser considerada é a redução da convivência familiar, já que os estudantes passam muito tempo deslocando-se entre a residência e a escola (MORAIS, 2018).

Tabela 2: Desempenho no Enem dos alunos do ensino médio do IFRR/CNP e IFRR/BV nos anos 2017 e 2018, em comparação com a média nacional.

\begin{tabular}{c|c|c}
\hline $\begin{array}{c}\text { Desempenho dos alunos do } \\
\text { IFRR/CNP } \\
\text { (Redação + questões objetivas) }\end{array}$ & $\begin{array}{c}\text { Desempenho dos alunos do } \\
\text { IFRR/BV } \\
\text { (Redação + questões } \\
\text { objetivas) }\end{array}$ & $\begin{array}{c}\text { Média Nacional } \\
\text { (Redação + questões } \\
\text { objetivas) }\end{array}$ \\
\hline \multicolumn{3}{|c|}{2017} \\
\hline 488,7 & 584,79 & 523,32 \\
\hline 498,39 & $\mathbf{2 0 1 8}$ & 529,64 \\
\hline
\end{tabular}

Fonte: Adaptado de INEP, 2017 e 2018.

A professora e pesquisadora do IFRR/CNP Alaine Andrade de Morais, em sua dissertação de mestrado entitulada "Contribuições ao planejamento da etapa técnico - econômica do transporte escolar rural", após analisar as rotas e o tempo gasto no transporte escolar rural dos estudantes do IFRR/CNP, fez algumas sugestões para diminuir o tempo de permanência dos alunos no translado de suas residências até a escola. As sugestões surgiram após a 
pesquisadora percorrer o caminho com os estudantes, cronometrando o tempo e medindo a quilometragem percorrida pelo veículo escolar na ida e na volta dos estudantes. Assim, foi possível verificar os aspectos a serem melhorados para que o tempo de deslocamento até a escola seja diminuído.

De acordo com a Tabela 1, o trecho mais longo percorrido pelos alunos (4h30min) é o que vai da cidade de Caracaraí-RR ao IFRR/CNP, com 226 quilômetros percorridos diariamente (ida e volta do instituto). Ao fazer esse trajeto com os alunos, Morais (2018) detectou 50 paradas no perímetro urbano da cidade de Caracaraí para embarque/desembarque de estudantes. Tal cenário resultou no aumento do tempo de permanência dos discentes dentro do ônibus em 1h30min diários (gastos apenas com paradas). Também foi verificado que o percurso total do ônibus escolar com as 50 paradas dentro da cidade correspondia a 16,46 km diários, ou seja, havia muita proximidade entre as paradas, além das dificuldades do veículo rodoviário de grande porte em transitar nas vias esburacadas e sem infraestrutura. Para contornar o problema, houve um melhor planejamento da rota do ônibus escolar e estabeleceu-se a distância mínima de 1,2 km entre as paradas. Tais medidas resultaram em uma redução do percurso interno de 16,46km para 9,14 km (aproximadamente 56\%) e em uma redução no tempo de permanência no veículo de 50\%, passando de 1h30min para 45 minutos no trecho urbano. Um fluxograma para facilitar as tomadas de decisões está representado na Figura 1.

Figura 1: Fluxograma de tomada de decisões para diminuir o tempo de deslocamento do transporte escolar.

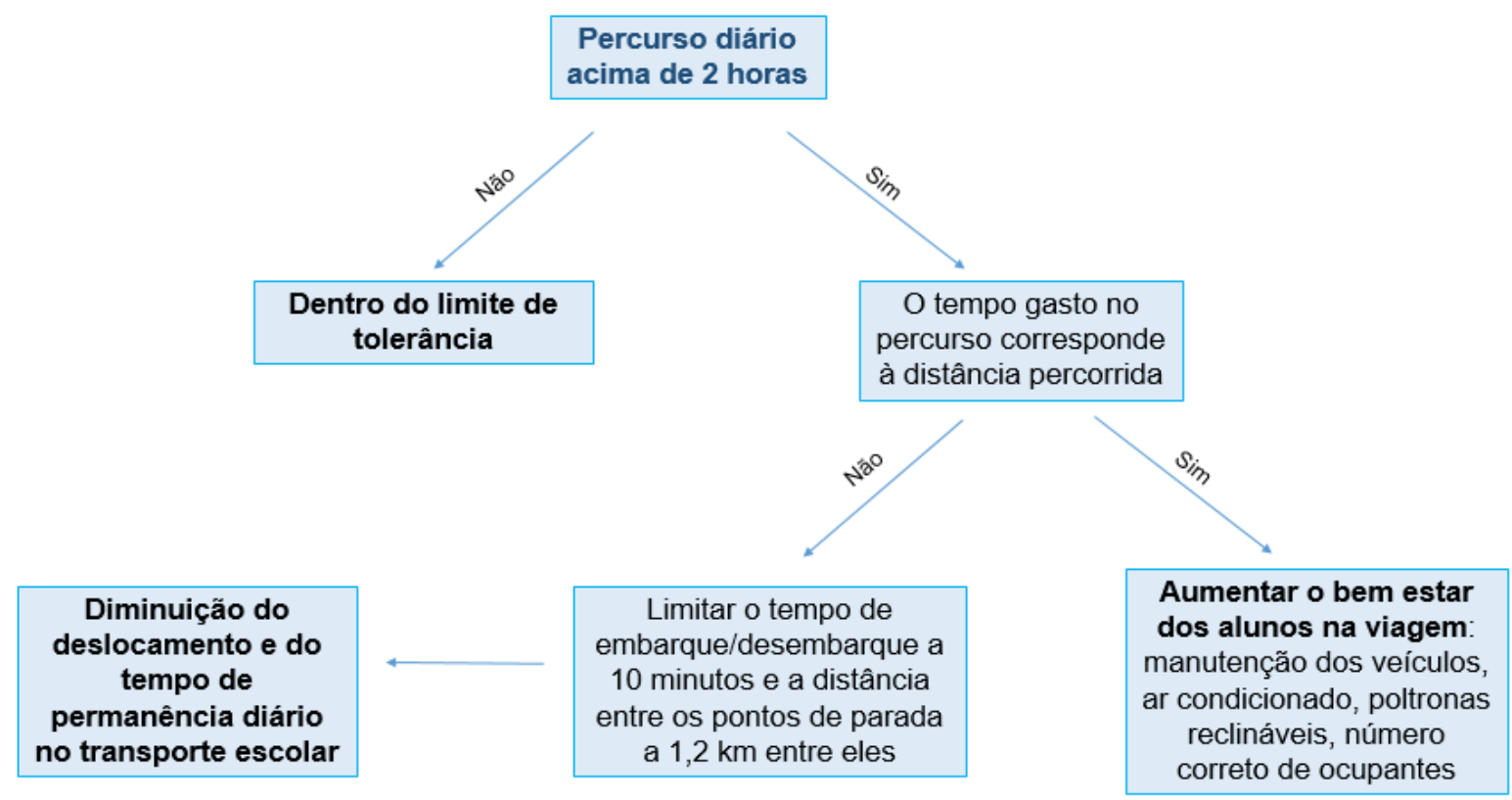

Fonte: Adaptado de Morais (2018). 
No fluxograma da Figura 1 acima, pode-se observar que, se o tempo for superior a duas horas diárias dentro do veículo, é necessário que se faça um estudo do percurso, para verificar se há paradas em excesso e um gasto de tempo acima do esperado em relação à quilometragem do trajeto. Se confirmada o gasto desnecessário de tempo, deve-se fazer um planejamento para embarque/desembarque dos estudantes (1,2 km entre as paradas) e, também, estipular o limite de dez minutos em cada ponto de parada do veículo. Caso o tempo, apesar de longo, estiver correspondendo a distância percorrida diariamente pelo transporte escolar (em caso de altas quilometragens no trajeto), deve-se então melhorar as condições do veículo, a fim de minimizar o desgaste dos estudantes e garantir um bom aproveitamento nas aulas.

A partir destes estudos, foi possível, também, criar um checklist de sugestões para diminuir o tempo de percurso do transporte escolar, sintetizado na Tabela 3.

Tabela 3: Checklist para planejamento do transporte rural.

$\checkmark$ Reduzir o tempo de embarque para no máximo dez minutos, como é comum nas plataformas rodoviárias;

$\checkmark$ Acompanhar e manter o controle nos percursos realizados pelos veículos alocados para 0 transporte rural dos alunos;

$\checkmark$ Observar as diferenças de quilometragem percorridas, a lotação dos ônibus e a observância dos tempos de percursos e dos pontos de paradas.

Fonte: Adaptado de Morais (2018).

\section{Considerações Finais}

É evidente que ao longo dos anos, o transporte escolar rural vem passando por melhorias através dos programas de financiamento do transporte escolar do governo. Porém, as longas distâncias a serem percorridas e a condição das estradas acabam aumentando o tempo de permanência dos alunos no transporte escolar rural, fato que influencia negativamente no rendimento escolar.

Para resolver esse problema, faz-se necessário um melhor planejamento por parte das escolas e empresas de transporte do trajeto a ser percorrido e dos pontos de parada para embarque de estudantes, a fim de minimizar 0 tempo gasto no percurso até a escola. Ao governo, tanto em esfera federal, quanto estadual e municipal, cabe a implementação de políticas públicas que proporcionem a melhoria das estradas e aumento da pavimentação em zonas 
rurais, além de instalação de pontos adequados de embarque e desembarque, garantindo assim transportes escolares adequados para docentes e estudantes das escolas rurais.

Por fim, por parte dos educadores e gestores educacionais, cabe a formulação das políticas educativas contextualizadas às especificidades do meio, valorizando a cultura e tendo como objetivo principal a oferta de uma educação de qualidade, que assegure a ampliação dos direitos de cidadania da população rural e garanta o direito do aluno ao acesso e permanência na escola e, sua inserção no mercado de trabalho.

\section{REFERÊNCIAS}

ARAÚJO, C. E. F. Análise de eficiência nos custos operacionais de rotas do transporte escolar rural. Dissertação de Mestrado em Transportes, Universidade de Brasília - UnB, DF, 2008.

CÂMARA, M. T; LOPES, E. P; MONTEIRO, T. Transporte escolar rural como instrumento de viabilização do acesso à educação: o que dizem as leis? Centro Interdisciplinar de Estudos em Transportes. Brasília-DF: Universidade de Brasília, 2008.

Centro de Formação de Recursos Humanos em Transportes - CEFTRU. Diagnóstico do transporte escolar rural. Volume I - Relatório Final. Centro Interdisciplinar de Estudos em Transportes, UnB, Brasília, DF, 2008.

EGAMI, C. Y.; SOUZA, R. F. A.; MAGALHÃES, M. T. Q.; COSTA, E. J. S. C.; ALVES, M. F. B.; YAMASHITA, Y. Panorama das políticas públicas do transporte escolar rural. Centro de Formação de Recursos Humanos em Transportes - CEFTRU, Universidade de Brasília - UnB, 2015.

FRIGATO, M. T. Política educacional rural: transporte escolar rural e escolarização. São Paulo, 2016.

GUIMARÃES, A. Por que o transporte escolar anda mal. Em Dia, Ed. 170, março/2004.

INSTITUTO NACIONAL DE ESTUDOS E PESQUISAS EDUCACIONAIS ANÍSIO TEIXEIRA - INEP. Microdados - Enem por escola. Brasília, DF: INEP, Ministério da Educação, 2017.

INSTITUTO NACIONAL DE ESTUDOS E PESQUISAS EDUCACIONAIS ANÍSIO TEIXEIRA - INEP. Microdados - Enem por escola. Brasília, DF: INEP, Ministério da Educação, 2018.

MARTINS, A. P. A. Análise dos impactos das condições do transporte escolar rural no rendimento escolar dos alunos. Dissertação de Mestrado - Programa de Pós-graduação em Transportes, Universidade de Brasília, 2010.

MARTINS, E. Contabilidade de custos. 9. ed. São Paulo: Atlas, 2006. 
MORAIS, A. A. Contribuições ao planejamento da etapa técnico - econômica do transporte escolar rural. Dissertação de Mestrado - Programa de Mestrado Profissional em Engenharia de Produção, Universidade Estadual Paulista (UNESP), campus Guaratinguetá, SP, 2018.

PEGORETTI, M. S. Definição de um indicador para avaliar a acessibilidade dos alunos da zona rural às escolas da zona urbana. Dissertação de Mestrado Programa de Pós-graduação em Engenharia Urbana, Universidade Federal de São Carlos, São Carlos, SP, 2005.

PEGORETTI, M. S.; SANCHES, S. P. Definição de um indicador para avaliar a acessibilidade dos alunos da zona rural às escolas da zona urbana. Revista Educação e Tecnologia, v.2, n.1, p.1-15, 2006.

SANCHES, S. P.; FERREIRA, M. A. G. Avaliação multicriterial de um sistema de transporte de alunos da zona rural. In: CONGRESSO DE PESQUISA E ENSINO EM TRANSPORTES, 2016, Brasília. Anais... Brasília: ANPET, v.2, fev. 2017. 